\title{
Adenocarcinomas of the upper third of the rectum and the rectosigmoid junction seem to have similar prognosis as colon cancers even without radiotherapy, SAKK 40/87
}

\author{
S. A. Käser • J. Froelicher • Q. Li • S. Müller • \\ U. Metzger • M. Castiglione • U. T. Laffer • C. A. Maurer
}

Received: 31 March 2014 / Accepted: 14 August 2014 /Published online: 28 August 2014

(C) Springer-Verlag Berlin Heidelberg 2014

\begin{abstract}
Purpose To investigate the prognosis of adenocarcinomas of the upper third of the rectum and the rectosigmoid-junction without radiotherapy.

Methods Patients from a multicenter randomized controlled trial from 1987-1993 on adjuvant chemotherapy for R0resected colorectal cancers with stage I-III disease were retrospectively allocated: cancers of the lower two-thirds of the rectum (11 cm or less from anal-verge, Group A, $n=205)$, of the upper-third of the rectum and rectosigmoid-junction $(>11-$ $20 \mathrm{~cm}$ from anal-verge, Group B, $n=142$ ), and of the colon ( $>20 \mathrm{~cm}$ from anal-verge, Group C, $n=378$ ). The total mesorectal excision (TME) technique had not been introduced yet. The adjuvant chemotherapy turned out to be ineffective. None of the patients received neoadjuvant or adjuvant radiotherapy.

Results The patients had a regular follow-up (median, 8.0 years). The 5 -year disease-free survival (DFS) rate was 0.54 (95\%CI, 0.47-0.60) in Group A, 0.68 (95\%CI, 0.60 0.75 ) in Group B, and 0.69 (95\%CI, 0.64-0.74) in Group C. The 5-year overall survival (OS) rate was $0.64(95 \% \mathrm{CI}, 0.57-$
\end{abstract}

The results of this study were presented at the annual meeting of the Swiss Surgical Society 2011, at the annual meeting of the Swiss Society of Gastroenterology and the Swiss Society of Visceral Surgery 2011, and at the annual meeting of the German, Austrian and Swiss Societies of Hemato-Oncology 2011.

S. A. Käser and J. Froelicher contributed equally.

S. A. Käser · J. Froelicher · Q. Li · S. Müller · U. Metzger •

M. Castiglione $\cdot$ U. T. Laffer $\cdot$ C. A. Maurer

For the Swiss Group for Clinical Cancer Research (SAKK),

Effingerstrasse 40, Bern 3008, Switzerland

C. A. Maurer $(\bowtie)$

Department of General, Visceral, Vascular and Thoracic Surgery,

Hospital of Baselland, Liestal, affiliated to the University of Basel,

Rheinstrasse 26, 4410 Liestal, Switzerland

e-mail: christoph.maurer@hin.ch
0.71) in Group A, 0.79 (95\%CI, 0.71-0.85) in Group B, and 0.77 (95\%CI, 0.73-0.81) in Group C. Compared with Group $\mathrm{C}$, patients in Group A had a significantly worse OS (hazard ratio [HR] for death 2.10) and a worse DFS (HR for relapse/ death 1.93), while patients in Group B had a similar OS (HR 1.12) and DFS (HR 1.07).

Conclusions Adenocarcinomas of the upper third of the rectum and the rectosigmoid-junction seem to have similar prognosis as colon cancers. Even for surgeons not familiar with the TME technique, preoperative radiotherapy may be avoided for most rectosigmoid cancers above $11 \mathrm{~cm}$ from anal-verge.

Keywords Rectal cancer · Upper third · Rectosigmoid junction $\cdot$ Radiotherapy $\cdot$ Total mesorectal excision

\section{Introduction}

For patients with rectal cancer, the introduction of total mesorectal excision (TME) led to a reduction of the local recurrence rate [1-3], seemed to improve survival if systemic disease was absent [3], and allowed the preservation of the male genital function better when compared with conventional rectal cancer surgery [4].

By adding preoperative short-term radiotherapy, the 10year cumulative incidence of local recurrence could be reduced further from $11 \%$ for surgery alone to $5 \%$ for radiotherapy plus surgery (number needed to treat, 17) in a randomized controlled multicenter trial [2]. Interestingly, overall survival did not differ between the group with and the group without preoperative radiotherapy [2].

In a subgroup analysis of $n=180$ of the non-irradiated patients, $24 \%$ had no completely removed mesorectum [5]. This had a significant impact on overall recurrence rate and on overall survival rate in these patients [5]. Thus, especially in 
non-irradiated patients, the oncosurgical quality seems to be of utmost importance.

It can be concluded that only few patients seem to benefit from radiotherapy, but all are subject to the risk of the correlated short- and long-term side effects [6] with low numbers needed to harm $(\mathrm{NNH})$. These are fecal incontinence $(\mathrm{NNH}$, 7) [7], (male) genital dysfunction $(\mathrm{NNH}, 4)$ [8], urinary incontinence $(\mathrm{NNH}, 6)$ [9], anal blood loss $(\mathrm{NNH}, 13)$ [7], and mucus loss $(\mathrm{NNH}, 8)$ [7]. Further research is needed to identify those patients who do not benefit from preoperative radiotherapy.

One field of research is on the value of magnetic resonance imaging (MRI)-based preoperative radiochemotherapy in patients with rectal carcinoma treated with TME $[10,11]$. Preoperative radiochemotherapy seems to be avoidable in $45 \%$ of patients with stage II or III disease, if high quality of TME is achieved and preoperative MRI predicts the minimum distance from tumor to the anticipated circumferential resection margin to be more than $1 \mathrm{~mm}[10]$.

Another field of research is on the value of preoperative radiochemotherapy for carcinomas of the upper third of the rectum. It has been claimed that preoperative radiochemotherapy was only justified for $\mathrm{T} 4$ cancers of the upper third of the rectum [12]. According to the available literature, it is questionable if cancers of the upper third of the rectum indeed should be treated as rectal cancers or rather as sigmoid cancers $[13,14]$.

The aim of the present retrospective study was to clarify any differences in prognosis between low/mid rectal cancer and high rectal/rectosigmoid junction cancer compared with colon cancer. To exclude two of the probably main confounding factors such as the surgeons' expertise/performance, as well as any perioperative radiotherapy, we retrospectively analyzed a population from a prospective randomized controlled multicenter trial on colorectal cancer patients originating before adoption of the TME technique and before adoption of radiotherapy for rectal cancer (SAKK 40/87) [15]. Thus, going back in time, this study might provide further insight on which patients with rectal cancer have a similar prognosis as patients with colon cancer and may not benefit from preoperative radiotherapy.

\section{Materials and methods}

Patients with potentially curable colorectal cancer, who were included in the SAKK 40/87 trial, a multicenter prospective randomized controlled trial on short-term perioperative chemotherapy with 5-fluorouracil and mitomycin C from 1987 to $1993(n=769)$ [15], were retrospectively analyzed.

The SAKK 40/87 trial had the following inclusion criteria: potentially curable colorectal cancer, age equal to or less than 75 years, Eastern Cooperative Oncology Group performance status [16] equal to or less than 2, radical en-bloc resection of the tumor (R0), normal renal and hepatic function, and UICC 1987 (International Union against Cancer) stage I, II, or III disease $[15,17]$. Exclusion criteria were the presence of distant metastases, pre-existing malignancies (with the exception of adequately treated in situ carcinoma of the cervix, or basal cell carcinoma of the skin), insulin-dependent diabetes mellitus, acute infections, portal vein thrombosis, leucopenia, thrombocytopenia, receipt of preoperative chemo- or radiation therapy, and planned postoperative radio- or immunotherapy [15].

Ethical review board approval and written informed consent from all the patients were obtained [15]. Patients were randomly assigned to either surgery alone (arm 1, 5 years disease-free survival (DFS), $65 \%$; 5 years OS, $72 \%$ ), to an immediate postoperative continuous portal vein chemo infusion (arm 2, 5 y DFS, $60 \%$; 5 years OS, $69 \%$ ), or to the same chemo infusion administered through a central venous catheter (arm 3, 5 years DFS, $64 \%$; 5 years OS, $74 \%$ ) [15]. Thus, the study did not show any improvement of either the DFS or the overall survival (OS) for the administered chemotherapy regimens in arm 2 or 3 [15].

From the $n=769$ patients included in the prospective SAKK $40 / 87$ trial, $n=44$ were excluded for different reasons (see Fig. 1), and $n=725$ were analyzed.

The distance of the distal border of the tumor to the anal verge was assessed intraoperatively or by rigid proctoscopy and recorded in centimeters in $88 \%$ of the patients. The tumors of the remaining $12 \%$ of patients were categorized into the three groups by other descriptions of the tumor site in the surgical reports (e.g., relation of the tumor site to the peritoneal reflection or relation of the tumor site to the rectosigmoid junction featured by the loss of the taeniae coli and the loss of appendices epiploicae or distal security margin in combination with the distance of the anastomosis from the anal verge or combination of several). The different measurement conditions were taken into account, with the awareness that a bowel specimen shrinks to about one half of its length if it is fixed in formalin without stretching [18].

The included patients $(n=725)$ were prospectively categorized into three groups according to the site of the distal border of the tumor. Cancers of the lower two thirds of the rectum (Group A, $11 \mathrm{~cm}$ or less from the anal verge, $n=205$ ), cancers of the upper third of the rectum and the rectosigmoid junction (Group B, between $>11 \mathrm{~cm}$ and $20 \mathrm{~cm}$ from the anal verge, $n=$ 142), and colon cancers (Group C, $>20 \mathrm{~cm}$ from the anal verge, $n=378$ ) as shown in Fig. 2. An overview of the present investigation is shown in Fig. 1. Details regarding the regular follow-up were published elsewhere [15]. Time of relapse was defined as the time point when recurrent disease was first diagnosed or, if later confirmed, when it was first suspected. Disease-free survival was defined as the interval from surgery to the date of relapse, or the detection of a second tumor, or 
Fig. 1 Overview of the methodology of the present study

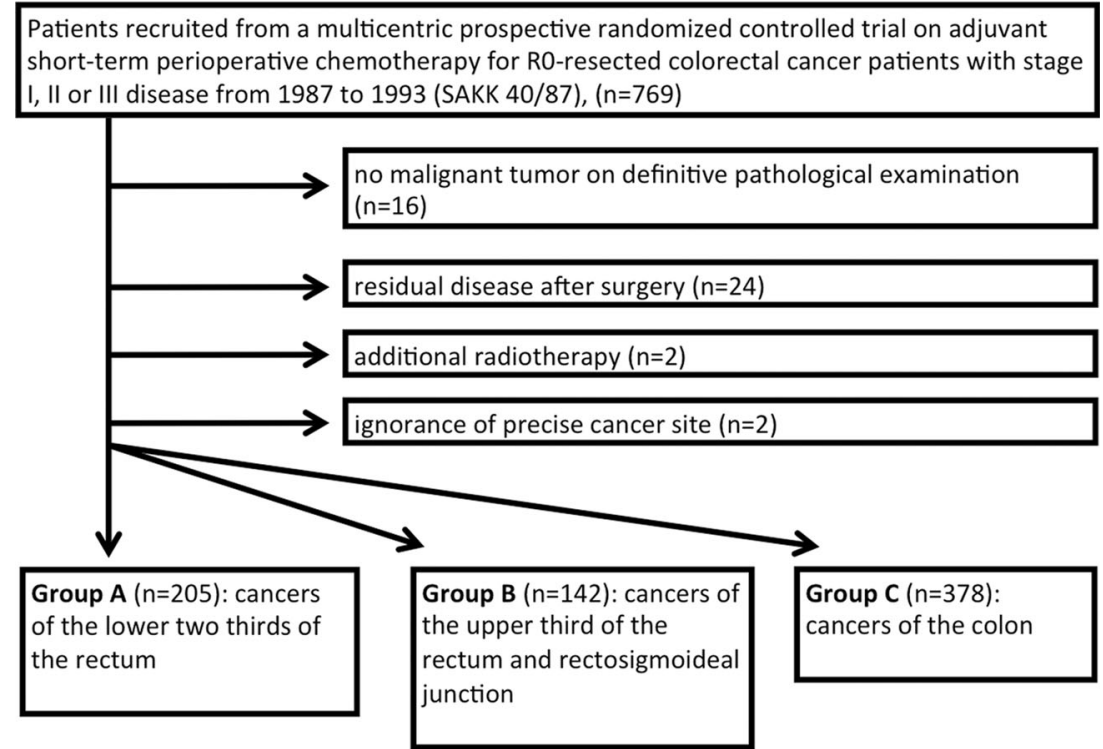

death, whichever occurred first [15]. The median follow-up time is 8.0 years.

\section{Statistics}

All the analyses were conducted using R 3.0.1 and SAS 9.2. All $P$ values are two-sided, with a significance level of 0.05 . As no adjustment for multiple testing was applied, the analyses are exploratory and hypothesis-generating. For categorical variables, chi-square tests were used to detect significant differences between groups. For numeric variables, KruskalWallis tests were used. Survival functions (for time-to-event variables) were estimated using Kaplan-Meier method, and median follow-up time was calculated by applying inverse Kaplan-Meier method. The confidence intervals of hazard ratios for Cox regression and survival function were

Fig. 2 Anatomy of the rectum, applied definitions of the rectum, and study group criteria

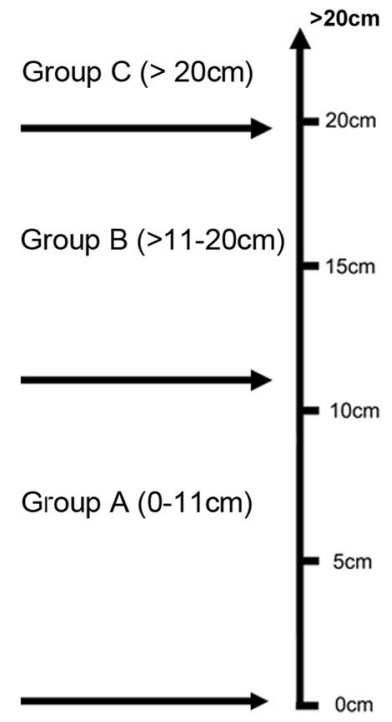

calculated based on cumulative hazard and point-wise logsurvival. $P$ values for the hazard ratios were calculated using Wald tests. Log-rank tests were applied to compare survival functions.

\section{Results}

The patient characteristics data and the data of surgical radicality and oncosurgical quality are shown in Table 1. The length of distal bowel margin in centimeters had no significant impact on neither OS $(p=0.391)$ or on DFS $(p=$ $0.100)$.

The 5-year DFS rate was $0.54(95 \% \mathrm{CI}, 0.47-0.60)$ in Group A, $0.68(95 \% \mathrm{CI}, 0.60-0.75)$ in Group B, and 0.69

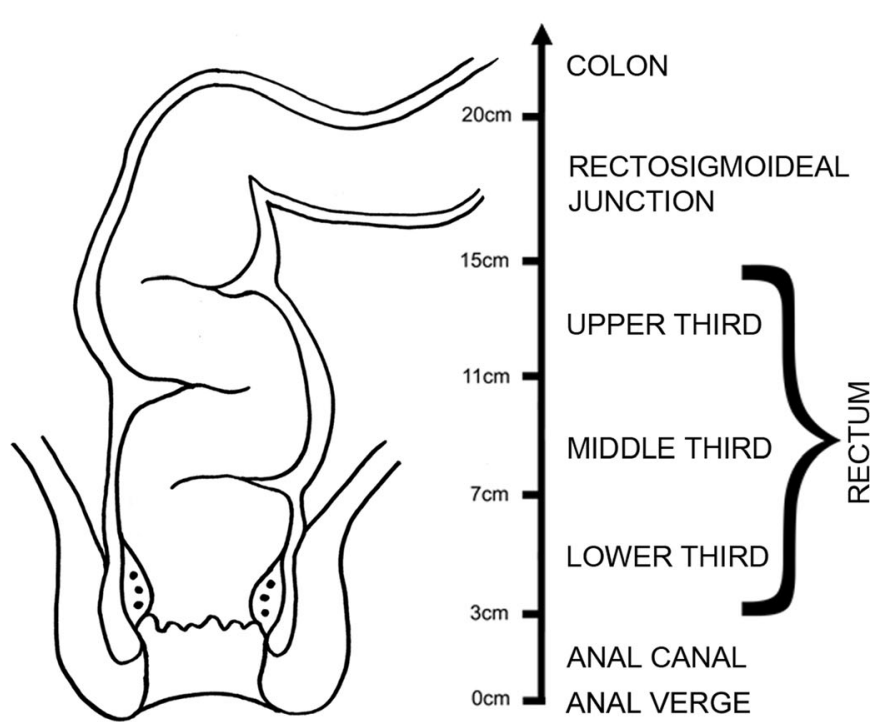


Table 1 Patient's characteristics and treatment (oncosurgical radicality/ quality and chemotherapy) of patients with cancer of the lower two thirds of the rectum (Group A), of the upper third of the rectum and the rectosigmoid junction (Group B), and of the colon (Group C); due to rounding, the sum does not always equal $100 \%$

\begin{tabular}{|c|c|c|c|c|}
\hline Variables & Group A $(n=205)$ & Group B $(n=142)$ & Group C $(n=378)$ & $p$ value \\
\hline Gender & & & & 0.332 \\
\hline Male & $125(61.0 \%)$ & $83(58.5 \%)$ & $207(54.8 \%)$ & \\
\hline Female & $80(39.0 \%)$ & $59(41.6 \%)$ & $171(45.2 \%)$ & \\
\hline Median age in years (range) & $62(15-76)$ & $64(37-75)$ & $65(18-75)$ & 0.152 \\
\hline Tumor stages $^{\mathrm{a}}$ (UICC 1987) & & & & $<0.001$ \\
\hline I & $71(34.6 \%)$ & $34(23.9 \%)$ & $62(16.4 \%)$ & \\
\hline II & $61(29.8 \%)$ & $54(38.0 \%)$ & $194(51.3 \%)$ & \\
\hline III & $71(34.6 \%)$ & $54(38.0 \%)$ & $121(32.0 \%)$ & \\
\hline$<$ Missing $>$ & $2(1.0 \%)$ & $0(0.0 \%)$ & $1(0.3 \%)$ & \\
\hline T stage & & & & $<0.001$ \\
\hline $\mathrm{T} 1$ & $13(6.4 \%)$ & $5(3.5 \%)$ & $26(6.9 \%)$ & \\
\hline $\mathrm{T} 2$ & $76(37.1 \%)$ & $38(26.8 \%)$ & $50(13.2 \%)$ & \\
\hline $\mathrm{T} 3$ & $113(55.12 \%)$ & $88(62.0 \%)$ & $275(72.8 \%)$ & \\
\hline $\mathrm{T} 4$ & $2(1.0 \%)$ & $11(7.8 \%)$ & $27(7.1 \%)$ & \\
\hline$<$ Missing $>$ & $1(0.5 \%)$ & $0(0.0 \%)$ & $0(0.0 \%)$ & \\
\hline Operation type & & & & $<0.001^{\mathrm{a}}$ \\
\hline Sphincter-sparing procedures & $132(64.4 \%)$ & $142(100.0 \%)$ & $378(100.0 \%)$ & \\
\hline Abdominoperineal excision & $73(35.6 \%)$ & $0(0.0 \%)$ & $0(0.0 \%)$ & \\
\hline Median number of examined nodes (range) & $8(0-36)$ & $9(0-36)$ & $10(0-61)$ & 0.103 \\
\hline Randomized treatment & & & & 0.945 \\
\hline Control & $70(34.2 \%)$ & $44(31.0 \%)$ & $127(33.6 \%)$ & \\
\hline Portal & $66(32.2 \%)$ & $47(33.1 \%)$ & $128(33.9 \%)$ & \\
\hline Systemic & $69(33.7 \%)$ & $51(35.9 \%)$ & $123(32.5 \%)$ & \\
\hline Days of hospital stay: median (range) & $18(1-136)$ & $15(6-62)$ & $14(2-124)$ & $<0.001$ \\
\hline 30-day mortality & $3(1.5 \%)$ & $5(3.5 \%)$ & $6(1.6 \%)$ & $0.204^{\mathrm{b}}$ \\
\hline
\end{tabular}

${ }^{a}$ Spearman's correlation coefficient between tumor stage and group is $0.072(p$ value $=0.053)$

${ }^{\mathrm{b}}$ Fisher's exact test extended with Monte-Carlo simulation

(95\%CI, 0.64-0.74) in Group C. The 5-year OS rate was 0.64 (95\%CI, 0.57-0.71) in Group A, 0.79 (95\%CI, 0.71-0.85) in Group B, and 0.77 (95\%CI, 0.73-0.81) in Group C. The causes of death are shown in Table 2. The corresponding Kaplan-Meier curves demonstrate the worse DFS and OS of group A, compared with group B and to group C (Figs. 3 and 4). Group A has significantly worse hazard ratios regarding time to local recurrence, time to distant recurrence, time to local and distant recurrence, and time to death due to tumor recurrence if compared with group $\mathrm{C}$ (Table 3). However, group B only had a significant worse hazard ratio for time to local recurrence if compared with group $\mathrm{C}$, while there were no significant differences between these groups regarding time to distant recurrence, time to local and distant recurrence, and time to death due to tumor recurrence (Table 3). Multivariate analysis with T-category and N-category as independent prognostic factors showed that group A had a significantly worse hazard ratio with a shorter DFS and a shorter OS if compared with group $\mathrm{C}$, while there was no significant difference in the hazard ratio for a shorter DFS or a shorter OS between groups B and C (Table 4).

Subgroup analysis comparing cancers of the upper third of the rectum $(>11-15 \mathrm{~cm}$ from anal verge) with cancers of the rectosigmoid junction $(>15-20 \mathrm{~cm}$ from anal verge) did not show significant differences regarding OS $(p=0.126)$ and DFS $(p=0.091)$.

\section{Discussion}

The aim of this study was to clarify the difference in prognosis between low/mid rectal cancer and high rectal/rectosigmoid junction cancer compared with colon cancer. Possible confounders such as effective preoperative or adjuvant radiochemotherapy or specialized surgery with adoption of TME were avoided.

Patients with cancers of the lower two thirds of the rectum had significantly more favorable tumor stages than patients 
Table 2 Causes of death of patients with cancer of the lower two thirds of the rectum (Group A), of the upper third of the rectum and the rectosigmoid junction (Group B), and of the colon (Group C)

\begin{tabular}{llll}
\hline $\begin{array}{l}\text { Variables } \\
\text { Survival status during study period }\end{array}$ & Group A $(n=205)$ & Group B $(n=142)$ & Group C $(n=378)$ \\
$\quad$ Alive & $105(51.2 \%)$ & $91(64.1 \%)$ & $259(68.5 \%)$ \\
$\quad$ Lost to follow-up & $5(2.4 \%)$ & $4(2.8 \%)$ & $11(2.9 \%)$ \\
$\quad$ Death & $95(46.3 \%)$ & $47(33.1 \%)$ & $108(28.6 \%)$ \\
Death causes & Group A $(n=95)$ & Group B $(n=47)$ & Group C (n=108) \\
Tumor recurrence & $74(77.9 \%)$ & $31(66.0 \%)$ & $64(59.2 \%)$ \\
Other causes (death not due to tumor or toxicity) & $16(16.8 \%)$ & $14(29.8 \%)$ & $30(27.8 \%)$ \\
Second malignancy & $1(1.1 \%)$ & $1(2.1 \%)$ & $9(8.3 \%)$ \\
Not known & $4(4.2 \%)$ & $1(2.1 \%)$ & $5(4.6 \%)$
\end{tabular}

Due to rounding, the sum does not equal the total percentage of deaths

${ }^{a}$ Metachronous primary colorectal neoplasia or neoplasia other than colorectal origin

with cancers of the upper third of the rectum, the rectosigmoid junction, and the colon as demonstrated in Table 1. Nevertheless the OS and DFS in patients with cancers of the upper third of the rectum and rectosigmoid junction were more favorable and very similar to colon cancer, whereas patients with cancers of the lower two thirds of the rectum had a much worse outcome.

The hazard ratio for local recurrence was significantly higher in patients with cancers of the upper third of the rectum and rectosigmoid junction compared with patients with colon cancer (demonstrated in Table 3). This suggests the necessity of neoadjuvant radiotherapy. However, first, no significant difference could be detected between these groups in multivariate analysis regarding DFS; second, the introduction of the TME technique after completion of enrollment of the patients of this study led to a relevant reduction of local recurrence
[1-3], and third, in randomized trials, preoperative shortcourse radiotherapy did not significantly reduce the local recurrence rate in patients with cancer of the upper rectum in conventional [19] and in TME surgery [20]. Thus, the detected difference in local recurrence is of limited relevance.

There are several possible reasons for the favorable prognosis of cancers of the upper third of the rectum and rectosigmoid junction compared with the much worse outcome of cancers of the lower two thirds of the rectum.

First: The surgical treatment of tumors of the upper third of the rectum and rectosigmoid junction is less challenging if compared with tumors of the lower two thirds of the rectum. In tumors of the upper third of the rectum and rectosigmoid junction, the same security margins are demanded as in cancers of the colon, and only partial mesorectal excision is required $[1,13]$. Furthermore, the operative situs is better
Fig. 3 Kaplan-Meier curves of the disease-free survival of patients with cancer of the lower two thirds of the rectum (Group A), of the upper third of the rectum, as well as the rectosigmoid junction (Group B), and of patients with colon cancer (Group C)

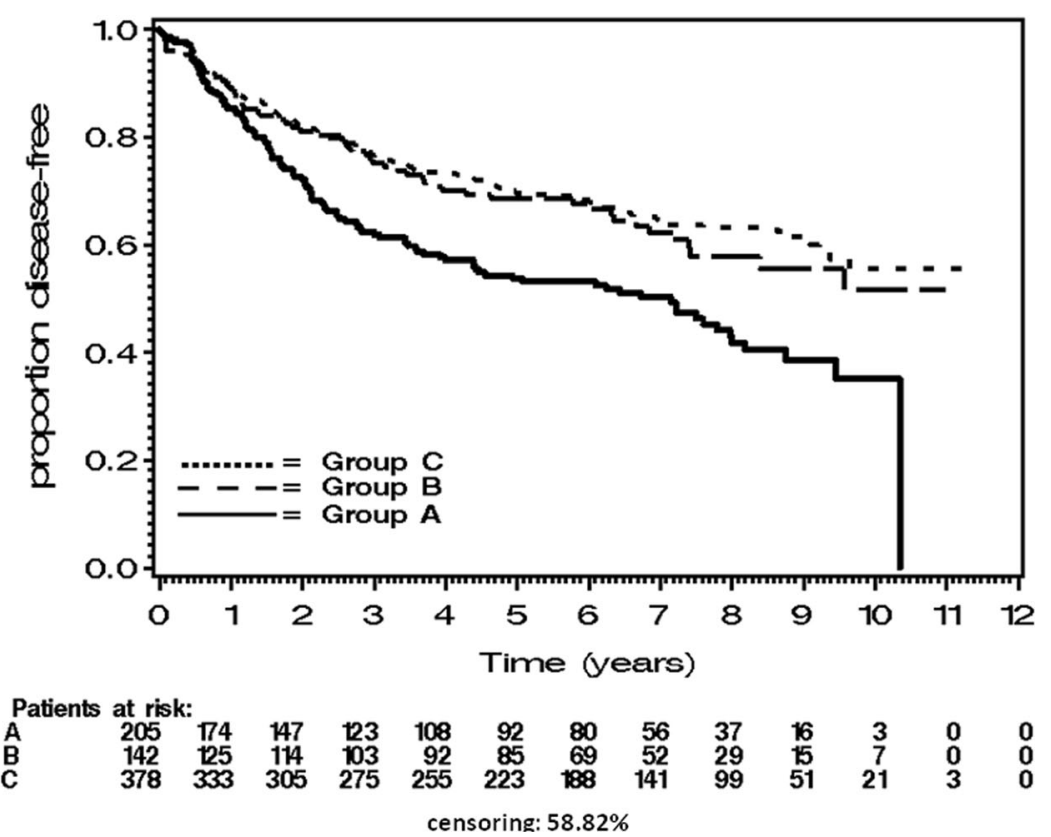


Fig. 4 Kaplan-Meier curves of the overall survival of patients with cancer of the lower two thirds of the rectum (Group A), of the upper third of the rectum, as well as the rectosigmoid junction (Group B), and of patients with colon cancer (Group C)

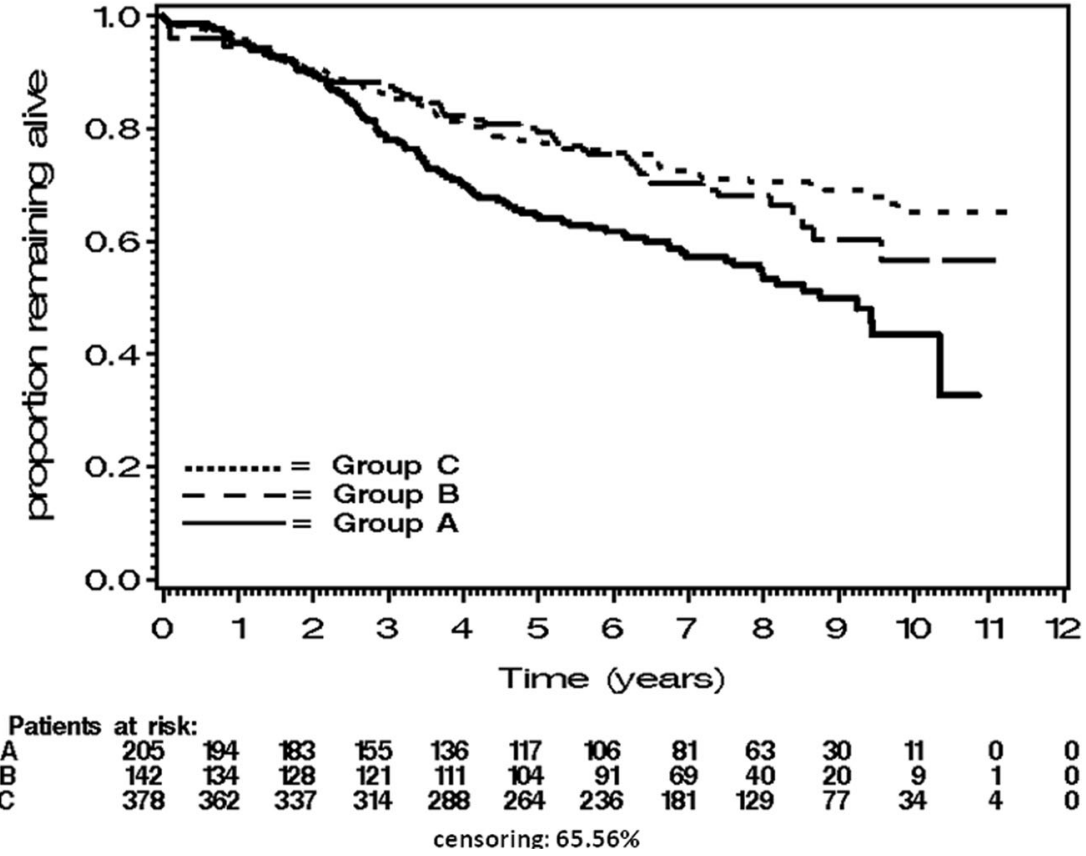

accessible if compared with the lower two thirds of the rectum with required TME and an often deep and narrow pelvis, especially in obese male patients with a bulky prostate.

Second: The mesorectum is more voluminous proximally, and the natural coning of the mesorectum distally (most of all ventrally) leads to a smaller circumferential margin, demanding a more exact dissection in the "holy plane" to avoid incomplete mesorectal excisions in tumors of the lower two thirds of the rectum. Especially in those tumors lying near the anal verge, required dissection is sometimes challenging.

Third: The different lymphatic anatomy of the upper third of the rectum is similar to the sigmoid colon, compared with the lower two thirds of the rectum [21]. The lymphatic drainage leads to a typical upward spread of cancers of the upper third of the rectum [22], which allows for a better local cancer control compared with cancers of the lower two thirds of the rectum. Cancers of the middle third of the rectum can spread laterally in the case of metastatic obstruction of mesorectal lymphatic tissue and cancers of the lower third of the rectum

Table 3 Comparison of death due to tumor recurrence and site of first recurrence between patients with cancer of the lower two thirds of the rectum (Group A) with patients with cancer of the upper third of the can spread up- and downward and laterally, even in early tumor stages [22].

Recently, it has been shown that the cranial border of the clinical target volume of short-course preoperative radiotherapy can safely be lowered for patients without expected nodal or circumferential resection margin involvement [23]. However, the acceptance that radiotherapy for rectal cancers of the proximal rectum can almost always be omitted is only slowly emerging. This might be due to a wide range of definitions of rectal cancer internationally and other factors. The macroscopic landmarks of the rectosigmoid junction (loss of the taeniae coli and appendices epiploicae with a wide variation between individuals) cannot be assessed before operation [24]. Therefore, the rectosigmoid junction in rectal cancer has been defined by the distance from the anal verge during rigid sigmoidoscopy of below $16 \mathrm{~cm}$ (UICC TNM classification [25]), of below $15 \mathrm{~cm}$ (most European studies [12]), and below $12 \mathrm{~cm}$ (in the United States of America [12]). Radiological landmarks (sacral promontory [26] or the S2/S3 rectum and the rectosigmoidal junction (Group B) with patients with cancer of the colon (Group C)

\begin{tabular}{|c|c|c|c|c|}
\hline & \multicolumn{2}{|l|}{ Group A vs. C } & \multicolumn{2}{|l|}{ Group B vs. C } \\
\hline & Hazard ratio $[95 \% \mathrm{CI}]$ & $p$ value & Hazard ratio $[95 \% \mathrm{CI}]$ & $p$ value \\
\hline Time to local recurrence & $9.00[4.53,17.89]$ & $<0.001$ & $4.33[1.97,9.55]$ & $<0.001$ \\
\hline Time to distant recurrence & $1.83[1.31,2.56]$ & $<0.001$ & $1.00[0.64,1.55]$ & 0.995 \\
\hline Time to local and distant recurrence & $2.30[1.69,3.11]$ & $<0.001$ & $1.27[0.86,1.87]$ & 0.238 \\
\hline Time to death due to tumor & $2.33[1.66,3.25]$ & $<0.001$ & $1.28[0.84,1.97]$ & 0.256 \\
\hline
\end{tabular}


Table 4 Results of Cox proportional hazard regression model comparing disease-free survival and overall survival of patients with cancer of the lower two thirds of the rectum (Group A) and of the upper third of the rectum as well as the rectosigmoid junction (Group B) to patients with colon cancer (Group C)

\begin{tabular}{lll}
\hline Variables & $\begin{array}{c}\text { Hazard ratio }[95 \% \mathrm{CI}] \\
\text { disease-free survival }\end{array}$ & $p$ value \\
Group A & $1.93[1.49,2.52]$ & $<0.001$ \\
Group B & $1.07[0.78,1.47]$ & 0.689 \\
Group C & $1.00[\mathrm{NA}]$ & Reference group \\
T1 & $0.37[0.17,0.82]$ & 0.014 \\
T2 & $0.60[0.36,1.02]$ & 0.058 \\
T3 & $0.78[0.49,1.23]$ & 0.284 \\
T4 & $1.00[\mathrm{NA}]$ & Reference group \\
Nodal positive & $2.16[1.70,2.73]$ & $<0.001$ \\
Nodal negative & $1.00[\mathrm{NA}]$ & Reference group \\
& Hazard ratio [95\% CI] & $p$ value \\
Group A & \multicolumn{1}{c}{ overall survival } & $<0.001$ \\
Group B & $1.10[1.57,2.79]$ & 0.517 \\
Group C & $1.00[\mathrm{NA}]$ & Reference group \\
T1 & $0.23[0.09,0.57]$ & 0.002 \\
T2 & $0.42[0.24,0.73]$ & 0.002 \\
T3 & $0.63[0.39,1.00]$ & 0.049 \\
T4 & $1.00[\mathrm{NA}]$ & Reference group \\
Nodal positive & $2.05[1.58,2.65]$ & $<0.001$ \\
Nodal negative & $1.00[\mathrm{NA}]$ & Reference group \\
\hline
\end{tabular}

$N A$ not applicable

vertebra [24]) used in the Japanese classification system [12] are not reliable alternatives to rigid sigmoidoscopy [27].

In our opinion, (preoperative) radiotherapy in cancers of the upper third of the rectum is still justified in the case of the tumor touching $(<=1 \mathrm{~mm})$ or infiltrating the mesorectal fascia and/or in the case of a cT4 tumor-infiltrating neighboring organs and making complete en bloc resection hazardous. However, in the case of limited infiltration of the fundus of the urinary bladder or infiltration of a segment of small bowel, complete en bloc resection is feasible and oncologically safe, and thus (preoperative) radiotherapy can be omitted.

The surgical radicality and oncosurgical quality was similar in the three groups as shown in Table 1 and cannot explain the observed differences.

It could be hypothesized that the favorable outcome of Group B is mainly due to inclusion of cancers of the rectosigmoid junction into this group. However, no significant differences regarding DFS and OS could be detected between patients with cancers of the upper third of the rectum $(>11-$ $15 \mathrm{~cm}$ from anal verge) and patients with cancers of the rectosigmoid junction ( $>15-20 \mathrm{~cm}$ from anal verge).

Due to the randomization of the study population, all the groups in the present study had a similar frequency of chemotherapy as shown in Table 1 . Furthermore, since chemotherapy - as given in this protocol - turned out to be ineffective, there is very little risk of bias due to different adjuvant therapies in this study.

\section{Limitations}

This is a retrospective analysis of data collected in a prospective randomized study, thus unknown bias is theoretically possible. However, according to the baseline data, the groups seem comparable (Table 1), and there was no significant difference between the groups regarding tumor stages and the type of (inefficient) adjuvant chemotherapy [15]. Therefore, a bias is rather unlikely.

The strength of this study is the study population originating from a time before adoption of the TME technique and before adoption of radiotherapy for rectal cancer (SAKK 40/ 87) [15]. This gives us the possibility to analyze which patients indeed have a benefit from radiotherapy and the TME technique. However, it has to be underlined that the outcome of the patients cannot be compared with the outcome of patients treated according to recent guidelines.

In $12 \%$ of the patients, the distance of the tumor to the anal verge was not exactly measured by rigid or flexible endoscopy (see "Methods" section). These patients were categorized into Group A, B, or C by other descriptions of the tumor site. However, the categorization was done before any statistical calculation was started. Thus, a bias due to migration between the groups is very unlikely.

\section{Conclusions}

Long-term results (OS, DFS) of patients with upper third rectal and rectosigmoid junction cancer seem very similar to those with colon cancer and much more favorable than those with lower two third rectal cancers. Adenocarcinomas of the upper third of the rectum and the rectosigmoid junction may therefore be oncologically regarded as colon cancers. Perioperative radiotherapy for these cancer sites may be omitted in most cases without compromising the oncological long-term result.

Authors' contributions Study conception and design (JF, QL, UM, MC, UTL, CAM)

Acquisition of data (JF, UM, MC, UTL, CAM)

Analysis and interpretation of data (SAK, JF, QL, SM, UM, MC, UTL, CAM)

Drafting of manuscript (SAK, JF, QL, CAM) CAM)

Critical revision of manuscript (SAK, JF, QL, SM, UM, MC, UTL,

Conflicts of interest None. 


\section{References}

1. Heald RJ, Ryall RD (1986) Recurrence and survival after total mesorectal excision for rectal cancer. Lancet 1(8496):1479-1482

2. van Gijn W, Marijnen CA, Nagtegaal ID, Kranenbarg EM, Putter H, Wiggers T, Rutten HJ, Pahlman L, Glimelius B, van de Velde CJ, Dutch Colorectal Cancer G (2011) Preoperative radiotherapy combined with total mesorectal excision for resectable rectal cancer: 12year follow-up of the multicentre, randomised controlled TME trial. Lancet Oncol 12(6):575-582. doi:10.1016/S1470-2045(11)70097-3

3. Maurer CA, Renzulli P, Kull C, Kaser SA, Mazzucchelli L, Ulrich A, Buchler MW (2011) The impact of the introduction of total mesorectal excision on local recurrence rate and survival in rectal cancer: long-term results. Ann Surg Oncol 18(7):1899-1906. doi:10. 1245/s10434-011-1571-0

4. Maurer CA, Z'Graggen K, Renzulli P, Schilling MK, Netzer P, Buchler MW (2001) Total mesorectal excision preserves male genital function compared with conventional rectal cancer surgery. Br J Surg 88(11):1501-1505. doi:10.1046/j.0007-1323.2001.01904.x

5. Nagtegaal ID, van de Velde CJ, van der Worp E, Kapiteijn E, Quirke P, van Krieken JH, Cooperative Clinical Investigators of the Dutch Colorectal Cancer G (2002) Macroscopic evaluation of rectal cancer resection specimen: clinical significance of the pathologist in quality control. J Clin Oncol: Off J Am Soc Clin Oncol 20(7):1729-1734

6. Junginger T, Maurer CA, Ruppert R (2013) Commentary on the editorial of Sautter-Bihl et al. in Strahlentherapie und Onkologie 2013 189:105-110. Strahlenther Onkol 189:697-699

7. Peeters KC, van de Velde CJ, Leer JW, Martijn H, Junggeburt JM, Kranenbarg EK, Steup WH, Wiggers T, Rutten HJ, Marijnen CA (2005) Late side effects of short-course preoperative radiotherapy combined with total mesorectal excision for rectal cancer: increased bowel dysfunction in irradiated patients - a Dutch colorectal cancer group study. J Clin Oncol: Off J Am Soc Clin Oncol 23(25):6199 6206. doi:10.1200/JCO.2005.14.779

8. Bonnel C, Parc YR, Pocard M, Dehni N, Caplin S, Parc R, Tiret E (2002) Effects of preoperative radiotherapy for primary resectable rectal adenocarcinoma on male sexual and urinary function. Dis Colon Rectum 45(7):934-939

9. Pollack J, Holm T, Cedermark B, Altman D, Holmstrom B, Glimelius B, Mellgren A (2006) Late adverse effects of shortcourse preoperative radiotherapy in rectal cancer. Br J Surg 93(12): 1519-1525. doi:10.1002/bjs.5525

10. Strassburg J, Ruppert R, Ptok H, Maurer C, Junginger T, Merkel S, Hermanek P (2011) MRI-based indications for neoadjuvant radiochemotherapy in rectal carcinoma: interim results of a prospective multicenter observational study. Ann Surg Oncol 18(10):2790-2799. doi:10.1245/s10434-011-1704-5

11. Ruppert R, Ptok H, Strassburg J, Maurer CA, Junginger T, Merkel S, Hermanek P (2012) [Quality indicators of diagnosis and therapy in MRI-based neoadjuvant radiochemotherapy for rectal cancer-interim analysis of a prospective multicentre observational study (OCUM).]. Zentralbl Chir. doi:10.1055/s-0031-1283922

12. Junginger T, Hermanek $P$ (2008) Problems in the treatment of upper rectal carcinoma. Der Chirurg; Zeitschrift fur alle Gebiete der operativen Medizin 79(4):327-339. doi:10.1007/s00104-008-1467-0
13. Lopez-Kostner F, Lavery IC, Hool GR, Rybicki LA, Fazio VW (1998) Total mesorectal excision is not necessary for cancers of the upper rectum. Surgery 124(4):612-617. doi:10.1067/msy.1998. 91361

14. Rosenberg R, Maak M, Schuster T, Becker K, Friess H, Gertler R (2010) Does a rectal cancer of the upper third behave more like a colon or a rectal cancer? Dis Colon Rectum 53(5):761-770. doi:10. 1007/DCR.0b013e3181cdb25a

15. Laffer U, Metzger U, Aeberhard P, Lorenz M, Harder F, Maibach R, Zuber M, Herrmann R (2008) Adjuvant perioperative portal vein or peripheral intravenous chemotherapy for potentially curative colorectal cancer: long-term results of a randomized controlled trial. Int J Color Dis 23(12):1233-1241. doi:10.1007/s00384-008-0543-8

16. Oken MM, Creech RH, Tormey DC, Horton J, Davis TE, McFadden ET, Carbone PP (1982) Toxicity and response criteria of the Eastern Cooperative Oncology Group. Am J Clin Oncol 5(6):649-655

17. Hermanek P, Sugg SL (1987) UICC. International union against cancer. TNM classification of malignant tumors. Springer-Verlag, Berlin

18. Kwok SP, Lau WY, Leung KL, Liew CT, Li AK (1996) Prospective analysis of the distal margin of clearance in anterior resection for rectal carcinoma. Br J Surg 83(7):969-972

19. Folkesson J, Birgisson H, Pahlman L, Cedermark B, Glimelius B, Gunnarsson U (2005) Swedish rectal cancer trial: long lasting benefits from radiotherapy on survival and local recurrence rate. J Clin Oncol 20(24):5644-5650

20. Kapiteijn E, Marijnen CA, Nagtegaal ID, Putter H, Steup WH, Wiggers T, Rutten HJ, Pahlman L, Glimelius B, van Krieken JH, Leer JW, van de Velde CJ (2001) Dutch colorectal cancer group: preoperative radiotherapy combined with total mesorectal excision for resectable rectal cancer. N Engl J Med 30(9):638-646

21. Maurer CA (2004) Colon cancer: resection standards. Tech Coloproctol 8(Suppl 1):s29-s32. doi:10.1007/s10151-004-0104-2

22. Steup WH, Moriya Y, van de Velde CJ (2002) Patterns of lymphatic spread in rectal cancer. A topographical analysis on lymph node metastases. Eur J Cancer 38(7):911-918

23. Nijkamp J, Kusters M, Beets-Tan RG, Martijn H, Beets GL, van de Velde CJ, Marijnen CA (2011) Three-dimensional analysis of recurrence patterns in rectal cancer: the cranial border in hypofractionated preoperative radiotherapy can be lowered. Int J Radiat Oncol Biol Phys 80(1):103-110. doi:10.1016/j.ijrobp.2010.01.046

24. Salerno G, Sinnatamby C, Branagan G, Daniels IR, Heald RJ, Moran BJ (2006) Defining the rectum: surgically, radiologically and anatomically. Color Dis: Off J Assoc Coloproctol Great Br Ireland 8(Suppl 3):5-9. doi:10.1111/j.1463-1318.2006.01062.x

25. Sobin LGM, Wittekind C (2009) UICC TNM classification of malignant tumours, 7th edn. John Wiley \& Sons, New York

26. Bagla N, Schofield JB (2007) Rectosigmoid tumours: should we continue sitting on the fence? Color Dis: Off J Assoc Coloproctol Great Br Ireland 9(7):606-608. doi:10.1111/j.1463-1318.2007. 01329.x

27. Baatrup G, Bolstad M, Mortensen JH (2009) Rigid sigmoidoscopy and MRI are not interchangeable in determining the position of rectal cancers. Eur J Surg Oncol: J Eur Soc Surg Oncol Br Assoc Surg Oncol 35(11):1169-1173. doi:10.1016/j.ejso.2009.02.004 